\title{
V14 Development and validation of multiplex test for measurement of antibodies against heptavalent vaccine componentes
}

Tamiris Azamor ${ }^{1}$, Luciana N. Tubarão ${ }^{1}$, Andréa M. V. da Silva ${ }^{1}$, Alessandro F. de Souza ${ }^{1}$, Jane da Silva ${ }^{1}$, Patrícia C. C. Neves ${ }^{1}$, Denise C. de S. Matos ${ }^{1}$

\section{${ }^{1}$ Bio-Manguinhos, Fiocruz, RJ}

Introduction: Multiplex is a liquid microarray assay that is replacing the immunoenzymatic assays (ELISA) in assessing the immunogenicity of multicomponent vaccines in pre-clinical and clinical trials. This technology utilizes fluorescent distinct microspheres as carriers for different molecules, allowing the simultaneous detection of multiple reactions in a small amount of samples and reagents with high reproducibility and sensitivity. The aim of this study is to develop and validate a multiplex assay for quantitation of antibodies against vaccine antigens present in current pentavalent vaccine (Difteric toxin, Tetanic toxin, $\mathrm{H}$. influenzae type $\mathrm{B}$ (Hib) polyssacharide (PRRP), B. pertussis and Hepatitis $B$ virus) besides the antigens intended to be used in na heptavalent formulation ( $\mathrm{N}$. Meningitidis type $\mathrm{C}$ conjugated and Poliovirus). During the upcoming introduction of the heptavalent vaccine in the National Immunization Program, this test might reduce the number of reactions to be performed during pre-clinical and clinical studies, generating working time optimization and lower costs for the National Health System of Brazil.

Objective: The aim of our study is to develop and validate a multiplex assay to quantify $\lg G$ against all antigens present in heptavalent vaccine formulation.

Methodology: For this purpose, we have constructed standard curves utilizing diphtheria toxin (Dtx), tetanus toxin (Ttx), capsular polysaccharide of Hib (fosforibosilribitol phosphate - PRRP) and pertussis toxin (Ptx). In addition, 10 serum samples were dosed in monoplex and multiplex assays.

Results: The results demonstrated a good performance of all those curves when comparing monoplex assay and multiplex assay. We have demonstrated that there is a high correlation between monoplex assay, multiplex assay and ELISA tests.

Conclusion: These results show the feasibility and applicability of this approach for clinical studies of combined vaccines.

Palavras-Chave: Multiplex, Heptavalent Vaccine 